\title{
Controlling precise movement with stochastic signals
}

\author{
Enrico Rossoni $\cdot$ Jing Kang $\cdot$ Jianfeng Feng
}

Received: 7 October 2009 / Accepted: 22 February 2010

(C) Springer-Verlag 2010

\begin{abstract}
In a noisy system, such as the nervous system, can movements be precisely controlled as experimentally demonstrated? We point out that the existing theory of motor control fails to provide viable solutions. However, by adopting a generalized approach to the nonconvex optimization problem with the Young measure theory, we show that a precise movement control is possible even with stochastic control signals. Numerical results clearly demonstrate that a considerable significant improvement of movement precisions is achieved. Our generalized approach proposes a new way to solve optimization problems in biological systems when a precise control is needed.
\end{abstract}

Keywords Neural control - Noncovnex optimization . Young measure $\cdot$ movement control

\section{Introduction}

The sheer complexity of movement control is often masked by the effortless ease with which we move our body, and only becomes evident when we try to build machines that perform similar tasks as we do. What makes the problem hard is the presence of uncertainty both in the external world, and in our own sensory-motor systems (Harris and Wolpert 1998; Osborne et al. 2005; Tanaka et al. 2006). Indeed, one of the

E. Rossoni · J. Kang $\cdot$ J. Feng $(\varangle)$

Department of Computer Science, University of Warwick,

Coventry CV4 7AL, UK

e-mail: jianfeng.feng@warwick.ac.uk

J. Feng

Centre for Computational System Biology, Fudan University, Shanghai, People's Republic of China

e-mail: jffeng@fudan.edu.cn central issues in Neuroscience is to explain how the nervous system deals so effectively with noise and variability.

The minimum-variance principle proposed by Harris and Wolpert (1998) in their seminal work has largely influenced the theoretical studies on the neural basis of motor control. There, the authors argued that the observed characteristics of our movements (e.g., the trajectories and velocity profiles of the eyes during a saccade or of the hand in a reaching task) are the end result of a process whereby the brain seeks to minimize the execution error (variance) caused by noise inherent in the neural control signals. Within such a framework, the actual control signals emitted by the nervous system would be the (approximately) optimal solutions to related stochastic control problems. In spite of its wide success, this theory still presents some unresolved issues.

One of its main conclusions is that, assuming that noise in the neural control signals is signal-dependent, and that the objective is to reduce the movement error, the optimal control signals are smooth function of time, as opposed to the degenerate, bang-bang controls that would result if the noise was independent of the signal. In Harris and Wolpert (1998), this result was obtained numerically under the specific assumption that the control signal is a stochastic process with an index of dispersion ${ }^{1}$ equal to one ( $\alpha=1$ in our setting, see Fig. 1 and "Model" section). Later, an analytical solution was found (Feng and Zhang 2002; Feng et al. 2004) that generalized this result to a whole range of noise models ( $\alpha \geq 0.5$ in our setting). However, for $\alpha<0.5$ the scenario changes radically due to lack of convexity of the

\footnotetext{
1 The index of dispersion is defined as half the power of the neural signal in this article. For example, let $\alpha$ denotes the index of dispersion, then the variance of the noise of the driving signal is expressed in the form of $k u_{t}^{2 \alpha}$, where $k$ is the scale and $u_{t}$ is the neural driving signal at time $t$. If the noise is Poisson, $\alpha=0.5$.
} 
related cost functional. In this case, the cost functional tends to favor brief pulses of increasingly large amplitude; hence, the optimal control signals turn out to be degenerate, i.e., delta functions. We remark that any pulse-like form of control which may be taken to approximate the optimal solution in this case will produce velocity profiles with abrupt acceleration and deceleration phases, which differ markedly from the bell-shaped profiles observed experimentally.

In the light of these results, one may draw the conclusion that large noise is necessary to smooth out our movements. However, in most in vivo experiments (including multi-electrode array recordings from our group; Horton et al. 2005; Christen et al. 2006) neurons appear to receive and emit spike trains which are at most as variable as Poisson processes, i.e., $\alpha<0.5$. Besides, one must take into account that in vivo recordings are performed in a highly nonstationary environment which could lead to significantly overestimate the irregularity of firing. In conclusion, one is left to wonder whether the noise regime considered in Harris and Wolpert's paper (1998) is relevant.

This is linked to another issue. As illustrated in Fig. 1a, for $\alpha \geq 0.5$ the movement error has a positive lower bound, which sets an unsurpassable limit to the precision of the movement. Yet recent experimental evidence (Osborne et al. 2005) has indicated that most of the movement error is due to inaccurate sensory estimates of the external parameters which define the task, so noise in the motor system may not, by itself, limit our ability to move precisely.

All these bring us to the fundamental question which we would like to address here: Is it possible to achieve a precise control with a stochastic signal? As we mentioned above, the answer has to be negative for $\alpha \geq 0.5$; hence, one must look further into the case $\alpha<0.5$ to find possible solutions. However, in this case the problem is much harder to tackle because the cost functional of our optimal control task is no longer convex.

Here, we will first see how one can construct suitably defined generalized solutions for the optimal control signal when the cost functional is nonconvex $(0<\alpha<0.5)$, inspired by the idea of Young measure theory (Young 1937, 1942; Valadier 1990; Hanson 2007). In terms of these solutions, we will demonstrate that the movement error can approach zero, thus achieving a precise movement control.

\section{Young measure}

For an optimization problem, suppose $I_{0} \in \mathbf{R}$ is the objective functional to be optimized on a set $\mathcal{U}$, where $\mathcal{U}$ is a set of functions. The optimization problem is to find $u^{*} \in \mathcal{U}$ such that

$$
I_{0}\left(u^{*}\right)=\inf _{\mathcal{U} \rightarrow \mathbf{R} \cup\{+\infty\}} I_{0}(u) \in \mathbf{R}
$$

If the objective functional $I_{0}(u)$ is of the form

$I_{0}(u)=\int_{\Gamma} F_{0}(x, u(x)) \mathrm{d} x$,

where $\Gamma \subset \mathbf{R}^{N}$ and

$F_{0}: \Gamma \times \mathbf{R}^{m} \rightarrow \mathbf{R} \cup\{+\infty\}$,

and the integrand $F_{0}$ is convex on $u$ (Fig. 1B), direct method of the Calculus of Variations is the common technique to solve this kind of questions. However, when there is lack of existence of the classical solutions to the optimization problem (1), Young measure approach can be applied.

In most of the cases of lack of solutions in optimal control, the essential reason is the oscillatory behavior of minimizing sequences $^{2}$ (Pedregal 1999). We will demonstrate oscillatory behavior of the control solution by the following example. Consider the functional

$I_{0}(\lambda)=\int_{0}^{1}\left[X(t)^{2}+\left(1-\lambda(t)^{2}\right)\right] \mathrm{d} t$,

where $\lambda$ is a measurable function from $[0,1]$ to $[-1,1]$ and $X$ satisfies $\mathrm{d} X / \mathrm{d} t=\lambda$ with boundary conditions $X(0)=0$, $X(1)=0$. There is no $\lambda^{*}$ such that $I_{0}\left(\lambda^{*}\right)=\inf I_{0}(\lambda)$. It is clear that the functions $\lambda_{n}(t)=\operatorname{sign}\left[\sin \left(2^{n+1} \pi t\right)\right]$ form a minimizing sequence which asymptotically minimize the cost for $n \rightarrow \infty$, but due to the increasingly rapid oscillations, the sequence $\left\{\lambda_{n}\right\}$ admits no ordinary limit (see Fig. 1e). $I_{0}\left(\lambda^{*}\right)=0$ is impossible for a single function.

The scenario described above is typical. To deal with the oscillatory behavior of the optimal solutions, Young measure was proposed by Young $(1937,1942)$ as a tool. The basic idea underlying the Young measure approach is simple: we enlarge the class of the competing functions in such a way that, when extended to this new class, our optimization problem always admits a solution. We can regard each competing function $u(x), x \in \Omega$, as a family of probability measures $v_{x}=\delta_{u(x)}$ considered as a mapping $\nu: \Omega \rightarrow \mathcal{M}$ where $\mathcal{M}$ represents the set of Radon measures ${ }^{3}$ supported in some appropriate euclidean space and $\delta$ stands for the Dirac mass. Any such mapping $v=\left\{v_{x}\right\}_{x \in \Omega}$ is called a Young measure or parametrized measure. The success of Young measure in the field of optimization is due to the fact that the cost functionals are integrals. The advantage of this approach is that Young measure furnishes a convenient way of dealing with optimal solutions paying attention only to those features that make a solution optimal and disregarding accidental properties (Pedregal 1999).

\footnotetext{
2 The minimizing sequences asymptotically minimize the cost but have no limit in ordinary sense.

${ }^{3}$ A Radon measure is a Borel measure that is finite on compact sets.
} 
Fig. 1 a Plotted is the optimal variance $I\left(\lambda^{*}\right)$ versus the noise parameter $\alpha$. Results are scaled so that the case of $\alpha=1$, corresponds to unity. b A convex cost function allows us to find the minimal solution, corresponding to the case of $\alpha>0.5$ in (a). c For a concave cost function, the minimal points are the boundary, corresponding to the case of $\alpha<0.5$ in (a). Hence, the constraint is usually violated. d A proper combination of the boundary solutions (toggling between the boundary) leads to a minimal solution, and satisfies the constraint as well. e $I_{0}(\lambda)$ for different $\lambda_{1}, \lambda_{2}$, and $\lambda_{4}$. We see that increasing the frequency of toggling will reduce the variance

\section{A}

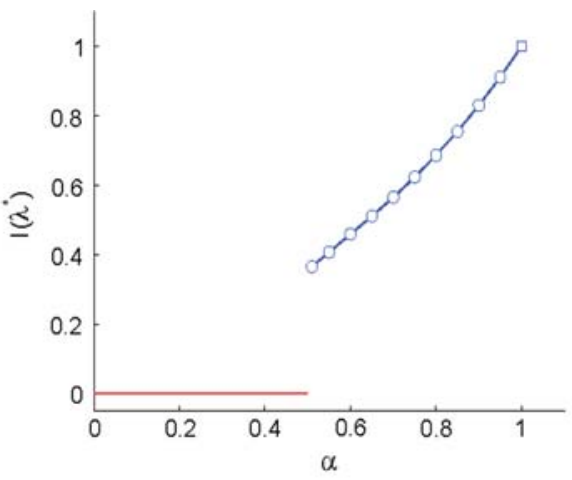

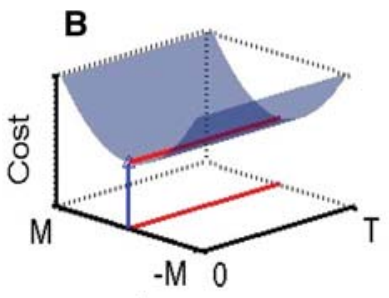

E $\lambda$

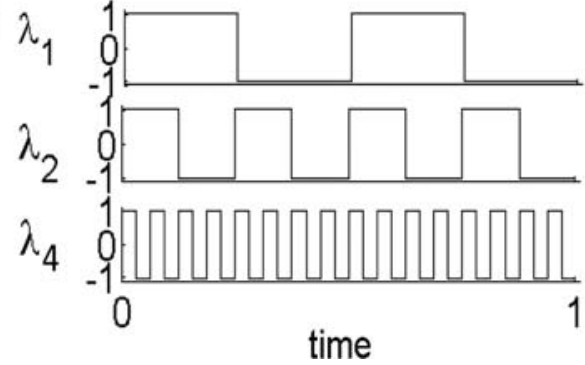

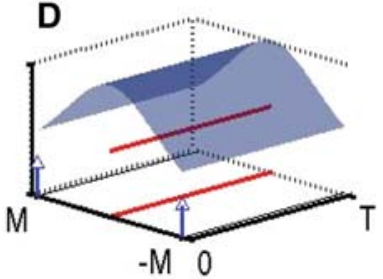
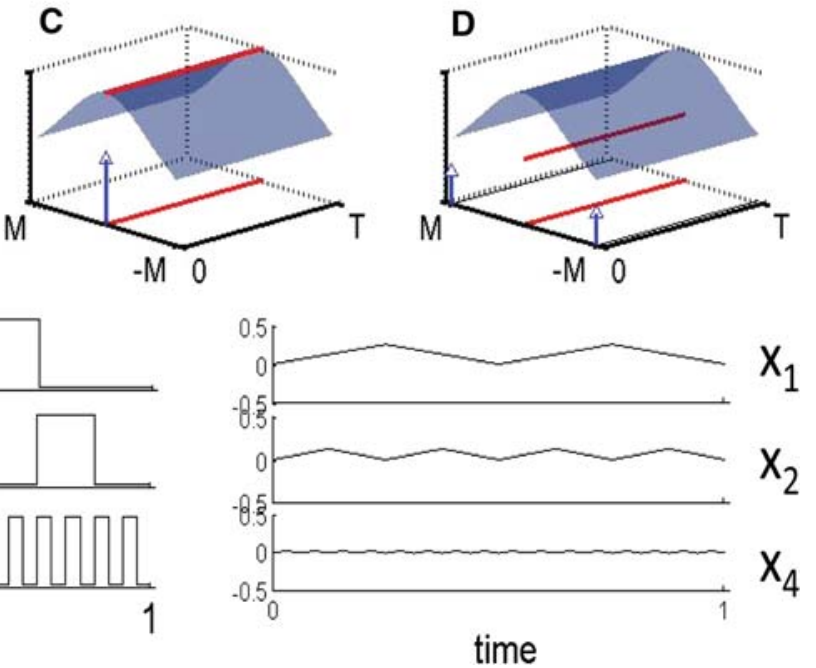

Originally introduced in the context of optimal control problems (Young 1937, 1942; Tuckwell 1984), Young measure has been successfully used in the field of engineering, material science, and partial differential equations (Valadier 1990). So far, Young measure have not yet been used in the field of biology, but it can be a convenient tool to solve some optimization problems in biological systems. The following sections provide examples illustrating the application of Young measure in motor control problems of saccadic eye movement.

\section{Example 1: saccadic eye movement model}

In order to illustrate our ideas, we consider a commonly used model of saccadic eye movements (Harris and Wolpert 1998; Robinson et al. 1986) which is simple enough to obtain analytical results. Let $x$ denote the (horizontal) eye displacement from the rest position measured in degrees (eccentricity). We consider saccades from the primary position $(x(0)=0)$ to targets located at a given eccentricity $(x(T)=D)$. We assume $x$ evolves in time according to

$\ddot{x}=-\frac{1}{\tau_{1} \tau_{2}} x-\frac{\tau_{1}+\tau_{2}}{\tau_{1} \tau_{2}} \dot{x}+\gamma[\lambda(t)+\xi(t)]$ where $\tau_{1}, \tau_{2}$, and $\gamma$ are parameters characteristic of the oculomotor plant. The driving term in brackets models the motor commands, i.e., the output of the motor neurons which innervate the extraocular muscles, ${ }^{4}$ and is assumed to be stochastic in nature. We separate a deterministic term $\lambda(t)$ (which we will denote as the control signal in the following) and a noise part $\xi(t)$, which is modeled as a mean zero, gaussian white noise with

$\mathrm{E}\left[\xi(t) \xi\left(t^{\prime}\right)\right]=\kappa|\lambda(t)|^{2 \alpha} \delta\left(t-t^{\prime}\right)$

where $\kappa$ and $\alpha>0$ are parameters. Equation 3 describes the experimental observation that the variability of neuronal signals in vivo tends to increase with the signal strength $\lambda(t)$, and generalizes the signal-dependent noise model considered by Harris and Wolpert (1998) where $\alpha=1$. We remark that the driving term in Eq. 2 is only a continuous approximation to the actual neural signal, which would be more suitably described as a stochastic point process (Tuckwell 1984; Brown et al. 1999; Feng and Tuckwell 2003; Feng

\footnotetext{
${ }^{4}$ We assume that force, or torque produced by the muscle is simply proportional to the neural signal. More refined models taking into account the temporal filtering property of the muscles, lead to higher-order systems.
} 
2004). In particular, for $\alpha<0.5, \alpha=0.5$, or $\alpha>0.5$, the input approximates of a process, respectively, less, equally, or more variable than a Poisson process.

Solving Eq. 2, we obtain solution $x(t)$ which is driven by $\lambda(t)$, with initial conditions $x(0)=0, \dot{x}(0)=0$ :

$$
\begin{aligned}
\left(\begin{array}{l}
x(t) \\
\dot{x}(t)
\end{array}\right)= & \left(\begin{array}{c}
\gamma \int_{0}^{t} b_{12}(t-s) \lambda(s) \mathrm{d} s \\
{ }_{0}^{t} \\
\gamma \int_{0}^{0} b_{22}(t-s) \lambda(s) \mathrm{d} s
\end{array}\right) \\
& +\left(\begin{array}{l}
\gamma \int_{0}^{t} b_{12}(t-s) \lambda^{\alpha}(s) \mathrm{d} B(s) \\
\gamma \int_{0}^{t} b_{22}(t-s) \lambda^{\alpha}(s) \mathrm{d} B(s)
\end{array}\right),
\end{aligned}
$$

where

$$
\left\{\begin{array}{l}
b_{12}(t)=\frac{\tau_{1} \tau_{2}}{\tau_{2}-\tau_{1}}\left[\exp \left(-\frac{t}{\tau_{2}}\right)-\exp \left(-\frac{t}{\tau_{1}}\right)\right] \\
b_{22}(t)=\frac{\tau_{1} \tau_{2}}{\tau_{2}-\tau_{1}}\left[\frac{1}{\tau_{1}} \exp \left(-\frac{t}{\tau_{1}}\right)-\frac{1}{\tau_{2}} \exp \left(-\frac{t}{\tau_{2}}\right)\right] .
\end{array}\right.
$$

and $B(t)$ stands for the standard Brownian motion.

Our optimal control problem is then defined as follows: For a target position $D$, and time $T, R>0$, find a control signal $\lambda^{*}(t)$ such that

$\mathrm{E}[x(t)]=D, \quad$ for $t \in[T, T+R]$

and

$$
\begin{aligned}
I\left(\lambda^{*}\right) & =\min _{\lambda \in \mathcal{L}^{2 \alpha}[0, T+R]} I(\lambda) \\
& =\min _{\lambda \in \mathcal{L}^{2 \alpha}[0, T+R]} \int_{T}^{T+R} \operatorname{Var}[x(t)] \mathrm{d} t .
\end{aligned}
$$

The space $\mathcal{L}^{2 \alpha}$ stands for $\mathcal{L}^{P}$ space, ${ }^{5}$ where $P=2 \alpha$. The physical meaning of the problem is clear: at time $T$, the eye must be on average on the target (Eq. 4), and as precisely as possible (Eq. 5). Also, the requirement that the average eye position be constant in the post-movement period $t \in$ $[T, T+R]$ implies that the average velocity must be zero on the target.

\footnotetext{
$\overline{5}$ The $\mathcal{L}^{P}$ space is defined as a set of all measurable functions from measurable space $\mathcal{S}$ to $\mathbb{C}$ or $\mathbb{R}$, whose $p$ th norm has a finite Lebesgue integral, i.e., $\|f\|_{p}=\left(\int_{\mathcal{S}}|f|^{p} d \mu\right)^{\frac{1}{p}}<\infty$
}

Note that

$$
\begin{aligned}
& \int_{T}^{T+R} \operatorname{Var}(x(t)) \mathrm{d} t \\
& =\gamma^{2}\left\langle\int_{T}^{T+R}\left[\int_{0}^{t} b_{12}(t-s) \lambda(s)^{\alpha} \cdot \mathrm{d} B(s)\right]^{2} \mathrm{~d} t\right\rangle \\
& =\gamma^{2} \int_{T}^{T+R}\left[\int_{0}^{t} b_{12}^{2}(t-s)|\lambda(s)|^{2 \alpha} \mathrm{d} s\right] \mathrm{d} t,
\end{aligned}
$$

then the original control problem defined by Eqs. 4 and 5 is reduced to the following optimization problem: find $\lambda^{*}(s) \in$ $\mathcal{L}^{2 \alpha}[0, T+R]$ which minimizes

$I(\lambda)=\int_{T}^{T+R}\left[\int_{0}^{t} b_{12}^{2}(t-s)|\lambda(s)|^{2 \alpha} \mathrm{d} s\right] \mathrm{d} t$

subject to the constraint

$$
\int_{0}^{t} b_{12}(t-s) \lambda(s) \mathrm{d} s=\frac{D}{\gamma}, \quad \text { for } t \in[T, T+R] .
$$

We can rewrite the above objective functional $I(\lambda)$ and express it by during-movement $\left(I_{1}(\lambda)\right)$ and post-movement $\left(I_{2}(\lambda)\right)$ functionals:

$I(\lambda)=I_{1}(\lambda)+I_{2}(\lambda)$,

where

$$
\begin{aligned}
& I_{1}(\lambda)=\int_{0}^{T}\left[\int_{T}^{T+R} b_{12}^{2}(t-s) \mathrm{d} t\right]|\lambda(s)|^{2 \alpha} \mathrm{d} s \\
& I_{2}(\lambda)=\int_{T}^{T+R}\left[\int_{T}^{s} b_{12}^{2}(t-s) \mathrm{d} t\right]|\lambda(s)|^{2 \alpha} \mathrm{d} s .
\end{aligned}
$$

When $2 \alpha>1$, the objective functional $I(\lambda)$ is convex, and we can solve this optimal control problem theoretically with the method Calculus of Variation. The detailed technique and solution is presented as follows.

3.1 Ordinary solution when $\alpha>0.5$

\subsubsection{Post-movement solution in $t \in[T, T+R]$}

The optimal post-movement solution $\lambda^{*}$ for $t \in[T, T+$ $R]$ of the objective functional $I_{2}\left(\lambda^{*}\right)$ is determined by the 
constraint (8) and its derivative directly. By differentiating Eq. 8 (since $R>0$ ), we obtain

$$
\begin{aligned}
& -\frac{1}{\tau_{2}} \exp \left(-\frac{t}{\tau_{2}}\right) \int_{0}^{t} \exp \left(\frac{s}{\tau_{2}}\right) \lambda(s) \mathrm{d} s+\frac{1}{\tau_{1}} \exp \left(-\frac{t}{\tau_{1}}\right) \\
& \int_{0}^{t} \exp \left(\frac{s}{\tau_{1}}\right) \lambda(s) \mathrm{d} s=0
\end{aligned}
$$

for $t \in[T, T+R]$. Solving Eqs. 8 and 10 we see that

$$
\left\{\begin{array}{l}
\int_{0}^{t} \exp \left(\frac{s}{\tau_{2}}\right) \lambda(s) \mathrm{d} s=\frac{D}{\tau_{1} \gamma} \exp \left(\frac{t}{\tau_{2}}\right) \\
\int_{0}^{t} \exp \left(\frac{s}{\tau_{1}}\right) \lambda(s) \mathrm{d} s=\frac{D}{\tau_{2} \gamma} \exp \left(\frac{t}{\tau_{1}}\right)
\end{array}\right.
$$

for $t \in[T, T+R]$. This implies

$\lambda^{*}(t)=\frac{D}{\tau_{1} \tau_{2} \gamma}, \quad \forall t \in[T, T+R]$

and in particular

$$
\left\{\begin{array}{l}
\int_{0}^{T} \exp \left(\frac{s}{\tau_{2}}\right) \lambda(s) \mathrm{d} s=\frac{D}{\tau_{1} \gamma} \exp \left(\frac{T}{\tau_{2}}\right) \\
\int_{0}^{T} \exp \left(\frac{s}{\tau_{1}}\right) \lambda(s) \mathrm{d} s=\frac{D}{\tau_{2} \gamma} \exp \left(\frac{T}{\tau_{1}}\right) .
\end{array}\right.
$$

\subsubsection{During-movement solution in $t \in[0, T]$}

To find the optimal signal $\lambda^{*}(t)$ in $t \in[0, T]$ for the objective functional $I_{1}(\lambda)$ during saccadic eye movement, we apply the calculus of variations method in (9). To this end, let us define

$$
\begin{aligned}
& \left\{\lambda, \int_{0}^{T} b_{12}(T-s) \lambda(s) \mathrm{d} s=\frac{D}{\gamma}, \lambda(t)=\frac{D}{\tau_{1} \tau_{2} \gamma},\right. \\
& t \in[T, T+R]\}=\mathcal{U}_{D} .
\end{aligned}
$$

For a small $\tau$, consider $\lambda+\tau \phi \in \mathcal{U}_{D}$, i.e.,

$$
\begin{gathered}
\phi \in\left\{\int_{0}^{T} \exp \left(\frac{s}{\tau_{1}}\right) \phi(s) \mathrm{d} s=0,\right. \\
\int_{0}^{T} \exp \left(\frac{s}{\tau_{2}}\right) \phi(s) \mathrm{d} s=0, \\
\phi(t)=0, t \in[T, T+R]\}=\mathcal{U}_{D}^{0} .
\end{gathered}
$$

The first two constraints in $\mathcal{U}_{D}^{0}$ are from Eq. (12). We then have

$$
\left.\frac{\mathrm{d} I_{1}(\lambda+\tau \phi)}{\mathrm{d} \tau}\right|_{\tau=0}=0,
$$

which gives

$$
\int_{0}^{T}\left\{\left[\int_{T}^{T+R} b_{12}^{2}(t-s) \mathrm{d} t\right]|\lambda(s)|^{2 \alpha-1} \operatorname{sgn}(\lambda(s)) \phi(s)\right\} \mathrm{d} s=0 .
$$

Comparing Eq. 15 with the first two constraints in $\mathcal{U}_{D}^{0}$, we conclude that

$$
\left[\int_{T}^{T+R} b_{12}^{2}(t-s) \mathrm{d} t\right]|\lambda(s)|^{2 \alpha-1} \operatorname{sgn}(\lambda(s))=A(\xi, \eta)
$$

almost surely for $s \in[0, T]$ and $A(\xi, \eta)$ with two parameters $\xi, \eta \in \mathbb{R}$ is of the form

$A(\xi, \eta)=\xi \exp \left(\frac{t}{\tau_{1}}\right)+\eta \exp \left(\frac{t}{\tau_{2}}\right)$,

being the solution of the following equations

$$
\left\{\begin{aligned}
\frac{D}{\tau_{1} \gamma} \exp \left(\frac{T}{\tau} 2\right)= & \int_{0}^{T} \exp \left(\frac{s}{\tau} 2\right) \cdot|A(\xi, \eta)|^{\frac{1}{2 \alpha-1}} \\
& \cdot \operatorname{sgn}[A(\xi, \eta)]\left(\int_{T}^{T+R} b_{12}^{2}(t-s) \mathrm{d} t\right)^{-\frac{1}{2 \alpha-1}} \mathrm{~d} s \\
\frac{D}{\tau_{2} \gamma} \exp \left(\frac{T}{\tau} 1\right)= & \int_{0}^{T} \exp \left(\frac{s}{\tau} 1\right) \cdot|A(\xi, \eta)|^{\frac{1}{2 \alpha-1}} \\
& \cdot \operatorname{sgn}[A(\xi, \eta)]\left(\int_{T}^{T+R} b_{12}^{2}(t-s) \mathrm{d} t\right)^{-\frac{1}{2 \alpha-1}} \mathrm{~d} s .
\end{aligned}\right.
$$

Therefore, for $\alpha>0.5$ we obtain

$$
\begin{aligned}
\lambda^{*}(t)= & \mid A(\xi, \eta)^{\frac{1}{2 \alpha-1}} \operatorname{sgn}[A(\xi, \eta)] \\
& \cdot\left(\int_{T}^{T+R} b_{12}^{2}(s-t) d s\right)^{-\frac{1}{2 \alpha-1}}
\end{aligned}
$$

for $t \in[0, T]$. Also, for the post-movement period $t \in$ $(T, T+R]$ the optimal solution is simply given by the holdon control $\lambda^{*}=\frac{D}{\tau_{1} \tau_{2} \gamma}$, as derived earlier. When $\alpha=0.5$, the solution is harder to find analytically, but similar conclusions hold. Hence, for $\alpha \geq 0.5$, a minimizer $\lambda^{*}$ is guaranteed to exist and to be unique by the convexity of the cost functional and the set of admissible controls. Finally, one can easily verify that $I\left(\lambda^{*}\right)>0$.

For $0<\alpha<0.5$ the cost functional is concave; therefore, according to Young measure theory, a solution, if exists, must 
be found among the extreme points of the set of admissible control $\Omega=\{\lambda \in \mathcal{L}$ : the constraint is verified $\}$ (Fig. 1c-d). This means that any solution must be a superposition of delta functions. For instance, one can easily verify that, for $t \in$ $[0, T]$, all controls of the form $\lambda(t)=\sum_{i} A_{i} \delta\left(t-t_{i}\right)$ with $t_{i} \in[0, T]$ and suitable choice of the constants $A_{i}$, will drive the system on the target with absolute precision, i.e., they bear a vanishing contribution to the error. ${ }^{6}$ We conclude, that for $0<\alpha<0.5$, the optimal control is degenerate and not unique, with $I\left(\lambda^{*}\right)=0$.

In Fig. 1a, we plotted the minimum movement error, $I\left(\lambda^{*}\right)$, as a function of $\alpha$. The meaning of this result is clear. For $\alpha \geq 0.5$ there is a lower bound to the movement error. In other words, although there exists an implementable and finite signal which minimizes the error, the end result will be degraded by higher noise levels in the system, consistent with our intuition. By contrast, for $0<\alpha<0.5$ the minimum error is zero; although, there is no finite control signal that can achieve it. The question we will address in the next section is whether-and most importantly how-such minimum can be approached.

\subsection{Generalized control when $\alpha<0.5$}

Based on our previous discussion, for $\alpha<0.5$ we can achieve an arbitrary degree of precision, although the optimal control $\lambda^{*}$ which would reduce the error to zero is not implementable. Obviously, if we could find a sequence of finite controls $\left\{\lambda_{M}^{*}\right\}$ satisfying

$$
\lim _{M \rightarrow \infty} I\left(\lambda_{M}^{*}\right)=0,
$$

we could use these as a replacement for $\lambda^{*}$ and improve the accuracy as we wished. For instance, one may consider selecting $\lambda_{M}^{*}$ among the minimizers of $I$ in the space of bounded controls

$\Omega_{M}=\Omega \wedge\left\{f \in \mathcal{L}^{2 \alpha}[0, T+R],|f| \leq M\right\}$.

Unfortunately this approach is not directly feasible in practice. Indeed, when the cost functional is not convex, there is no guarantee that the minimizers $\lambda_{M}^{*}$ exist among ordinary functions.

From the previous discussion on Young measure in Sect. 2, we can construct a generalized control for this nonconvex optimization problem. Particularly, this generalized control is a one-parameter family of probability distributions over the control domain indexed by time, i.e., $\tilde{v}=\left\{\tilde{v}_{t}\right\}_{t \in I}$. In other words, while an ordinary control is a mapping which assigns to each time a precise value to the driving signal, a

${ }^{6}$ For $t>T$, one could consider solutions of the form $\int_{T}^{T+R} \delta\left(t-t^{\prime}\right)$ $A\left(t^{\prime}\right) \mathrm{d} t^{\prime}$ generalized control provides at each time a probability distribution over all the allowed control values. One sees that ordinary controls map naturally onto (or can be identified with) a subset of generalized controls, ${ }^{7}$ hence the latter provides an extension of the former in some sense.

Let us see how our control problem can be reformulated here. For convenience of notation, we will represent this generalized control $\tilde{v}$ by a stochastic process $v(t)$ such that $P(v(t) \in A)=\tilde{v}_{t}(A)$ for any subset $A \in[0, T] .^{8}$ Then, for a generalized control $\tilde{v}$ we define the functional

$$
\begin{aligned}
\tilde{I}(\tilde{v}) & \doteq \mathrm{E} I(v) \\
& = \begin{cases}\int_{0}^{T}\left[\int_{T}^{T+R} b_{12}^{2}(t-s) \mathrm{d} t\right] \mathrm{E}\left(|v(s)|^{2 \alpha}\right) d s & s \in[0, T] \\
\int_{T}^{T+R}\left[\int_{T}^{s} b_{12}^{2}(t-s) \mathrm{d} t\right] \mathrm{E}\left(|v(s)|^{2 \alpha}\right) \mathrm{d} s & s \in[T, T+R],\end{cases}
\end{aligned}
$$

where $\mathrm{E}(\cdot)$ denotes the expectation with respect to the measure of the process $v(t)$. Finally, the constraints on the admissible generalized control are obtained in a similar fashion, i.e.

$$
\left\{\begin{array}{l}
\int_{0}^{t} \exp \left(\frac{s}{\tau_{2}}\right) E(v(s)) \mathrm{d} s=\frac{D}{\tau_{1} \gamma} \exp \left(\frac{t}{\tau_{2}}\right) \\
\int_{0}^{t} \exp \left(\frac{s}{\tau_{1}}\right) E(v(s)) \mathrm{d} s=\frac{D}{\tau_{2} \gamma} \exp \left(\frac{t}{\tau_{1}}\right)
\end{array}\right.
$$

for $t \in[T, T+R]$.

We are now in a position to reconsider our original problem in its generalized formulation. Starting from any ordinary control, we can thus consider all the generalized controls whose average correspond to it. In particular, when the cost functional is concave, any generalized control which is not exclusively concentrated on the signal, will bring a lower generalized cost, and the cost will be minimum for those measures which are concentrated on the extreme control values, say $\{0, M\}$. Thus, for any ordinary controls which obeys our problem constraints, we obtain a corresponding "optimal" generalized control in the form

$\tilde{v}_{t}=(1-\beta(t)) \delta_{0}+\beta(t) \delta_{M}$,

with $\beta(t) \in[0,1]$ and $\delta$ as the delta function.

By this, we proved that it is relatively easy in our case to find good, albeit perhaps not optimal, generalized solutions. These class of solutions are now defined in terms of a

\footnotetext{
$\overline{7}$ Every ordinary control $\lambda$ can be identified with its associated generalized control $\tilde{\lambda}_{t}=\delta_{\lambda(t)}$ where $\delta$ stands for the Dirac mass.

${ }^{8}$ However, we remark that the stochastic process $v$ here is only a formal "handle" to the underlying generalized control, and that strictly speaking, there are no "realizations" of $\tilde{v}$, since the latter does not take values in $U$.
} 
simple function, $\beta(t)$ and may be identified with a stochastic processes toggling between two values, i.e., $v$ such that $v(t) \in\{0, M\}$, with $P(v(t)=M)=\beta(t)$.

\subsubsection{Generalized post-movement solution in $t \in[T, T+R]$}

To minimize the post-movement objective functional $I_{2}(\lambda)$ with constraint (8) for $t \in[T, T+R]$, we restrict ourselves to consider generalized controls which are concentrated on extreme values only. To this end, consider the stochastic process $v(t), t \in[T, T+R]$, where $v(t) \in\{0, M\}$ and $P(v(t)=$ $M)=\beta(t) \in[0,1]$. Let us see the implications of our construction:

$$
\begin{aligned}
\mathrm{E}_{2}(v) & =\int_{T}^{T+R}\left[\int_{T}^{s} b_{12}^{2}(t-s) \mathrm{d} t\right] \mathrm{E}\left[|v(s)|^{2 \alpha}\right] \mathrm{d} s \\
& =\int_{T}^{T+R}\left[\int_{T}^{s} b_{12}^{2}(t-s) \mathrm{d} t\right] M^{2 \alpha} \beta(s) \mathrm{d} s
\end{aligned}
$$

with the constraints

$$
\left\{\begin{aligned}
\int_{0}^{t} \exp \left(\frac{s}{\tau_{2}}\right) E[v(s)] \mathrm{d} s & =\int_{0}^{t} \exp \left(\frac{s}{\tau_{2}}\right) M \beta(s) \mathrm{d} s \\
& =\frac{D}{\tau_{1} \gamma} \exp \left(\frac{t}{\tau_{2}}\right) \\
\int_{0}^{t} \exp \left(\frac{s}{\tau_{1}}\right) E[v(s)] \mathrm{d} s & =\int_{0}^{t} \exp \left(\frac{s}{\tau_{1}}\right) M \beta(s) \mathrm{d} s \\
& =\frac{D}{\tau_{2} \gamma} \exp \left(\frac{t}{\tau_{1}}\right)
\end{aligned}\right.
$$

for $t \in[T, T+R]$. The constraints equations above imply that $\beta(s)$ is a constant measure, independent of $s$ and

$\beta(s)=\frac{D}{M \tau_{1} \tau_{2} \gamma}$

for $s \in[T, T+R]$ and $M \gg 1$.

To further explore the advantages of our approach here, let us estimate the term $E I_{2}(v)$ in more details. Summarizing results above we have

$E I_{2}(\nu)=C_{2}(T, R) \cdot \frac{D}{M^{1-2 \alpha} \tau_{1} \tau_{2} \gamma}$

where

$C_{2}(T, R)=\int_{T}^{T+R}\left[\int_{T}^{s} b_{12}^{2}(t-s) d t\right] \mathrm{d} s$

is a constant depending on $T$ and $R$. Therefore, the variance goes to zero as $M$ goes to infinity at a rate of $1 / M^{1-2 \alpha}$ for $\alpha<1 / 2$. In other words, the smaller the $\alpha$ is (the less noisy the system is), the faster the variance approaches zero.
Numerically, the generalized control solution $\lambda_{M}^{*}(t)$ for $t \in[T, T+R]$ can be constructed in the following way. For a given time step $h$ and $t+(k+1) h \leq T+R, k=0,1,2, \ldots$, we define

$\lambda_{M}^{*}(t)=\left\{\begin{array}{l}M \text { if } 0 \leq t-T-k h<h * \beta(T+k h) \\ 0 \text { if } h * \beta(T+k h) \leq t-T-k h<h\end{array}\right.$

Therefore, $\lambda_{M}^{*}(t)$ is a pulse function of width $\beta(t)$. Obviously, when $h \rightarrow 0, \lambda_{M}^{*}(t) \sim v(t)$ for $t \in[T, T+R]$.

\subsubsection{Generalized during-movement solution in $t \in[0, T]$}

The same idea can be applied to finding the generalized solution in $[0, T]$ as well. The problem we consider here is to minimize the objective functional during movement $I_{1}\left(\lambda^{*}\right)$ with constraints (8) for $\lambda \in \mathcal{L}^{2 \alpha}[0, T+R] \wedge[-M, M]$. It is easily seen that $\nu(t)$ should be a random process taking values in $\{-M, 0, M\}$. To simplify the issue further, we only consider a stochastic process $v(t)$ taking two values alternatively. Denote $E=\{t, v(t) \geq 0\}$ and define $P(v(t)=M)=w(t)$ for $t \in E$, for $t \in[0, T]-E$, we have $P(\nu(t)=-M)=$ $w(t)$. Hence the process $v(t)$ is uniquely defined by a onedimensional function $w(t)$.

Define $\beta(t)=\left[w(t) I_{E}-w(t) I_{[0, T]-E}\right]$ the optimal problem becomes to find $w(t)$ to minimize

$I_{1}=\int_{0}^{T}\left[\int_{T}^{T+R} b_{12}^{2}(t-s) \mathrm{d} t\right] M^{2 \alpha}|\beta(s)| \mathrm{d} s$

with constraints

$$
\left\{\begin{array}{l}
\int_{0}^{T} \exp \left(\frac{s}{\tau_{2}}\right) \beta(s) \mathrm{d} s=\frac{D}{M \tau_{1} \gamma} \exp \left(\frac{T}{\tau_{2}}\right) \\
\int_{0}^{T} \exp \left(\frac{s}{\tau_{1}}\right) \beta(s) \mathrm{d} s=\frac{D}{M \tau_{2} \gamma} \exp \left(\frac{T}{\tau_{1}}\right)
\end{array}\right.
$$

for $|\beta(t)| \leq 1$.

To obtain the generalized control signal which asymptotically approaches the global minimum, we define

$\beta(t)=\frac{\xi_{1}}{\tau_{1}} \exp \left(\frac{t-T}{\tau_{1}}\right)+\frac{\xi_{2}}{\tau_{2}} \exp \left(\frac{t-T}{\tau_{2}}\right)$,

where $\xi_{1}, \xi_{2}$ are two constants to be determined later. For simplicity, let us further define $\omega_{i}(t)=\frac{1}{\tau_{i}}\left[\exp \left(\frac{t-T}{\tau_{i}}\right)\right]$ for $i=1,2$. The definitions above and the constraints yield

$$
\left\{\begin{array}{l}
\left\|\omega_{1}\right\|^{2} \xi_{1}+\left\langle\omega_{1}, \omega_{2}\right\rangle \xi_{2}=\frac{D}{M \tau_{1} \tau_{2} \gamma} \\
\left\langle\omega_{1}, \omega_{2}\right\rangle \xi_{1}+\left\|\omega_{2}\right\|^{2} \xi_{2}=\frac{D}{M \tau_{1} \tau_{2} \gamma}
\end{array}\right.
$$



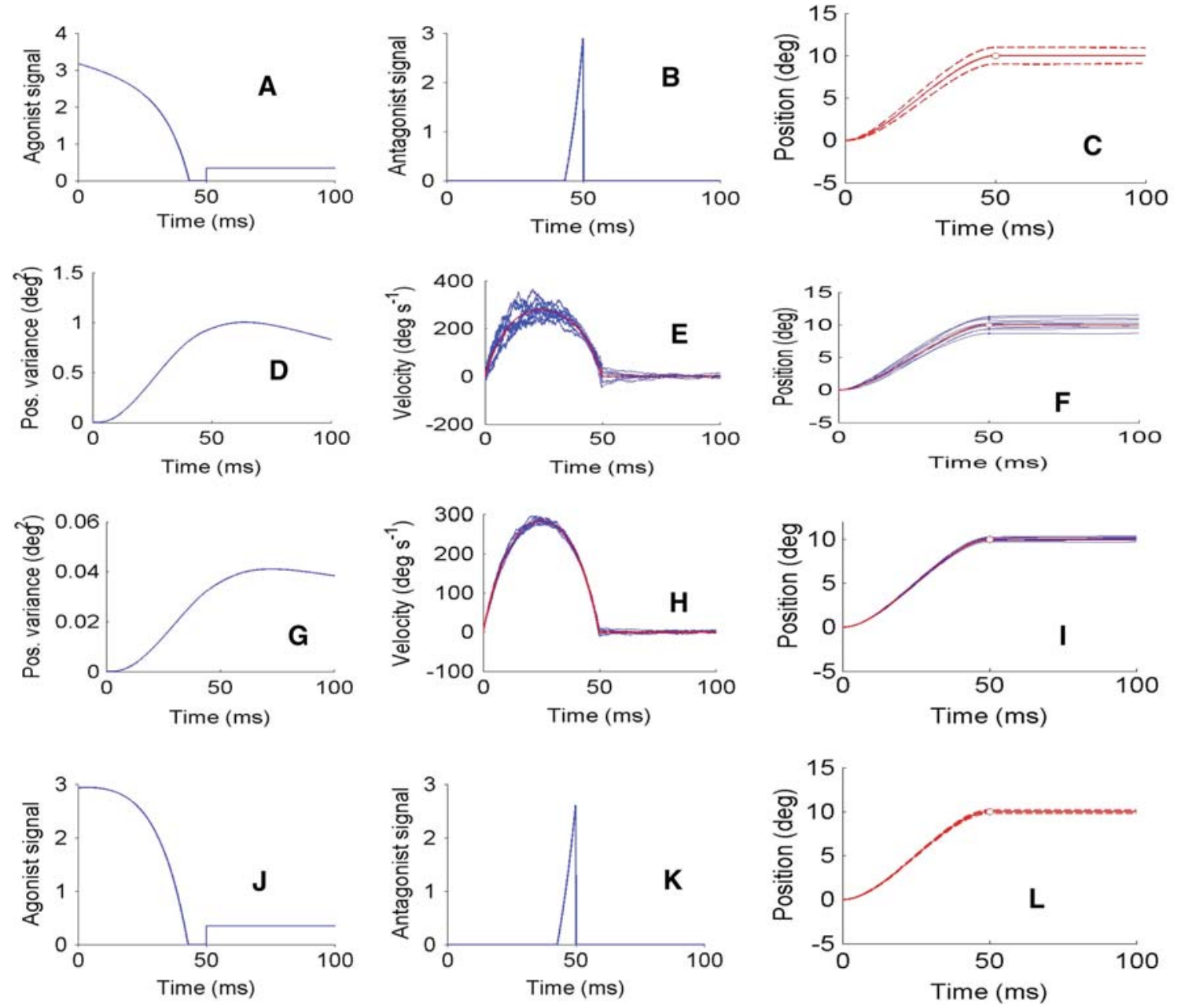

Fig. 2 Simulation results for saccadic eye movement model. $\mathbf{a}, \mathbf{b}, \mathbf{c}, \mathbf{d}, \mathbf{e}, \mathbf{f}$ are the case for $\alpha=1$ and $\mathbf{g}, \mathbf{h}, \mathbf{i}, \mathbf{j}, \mathbf{k}, \mathbf{l}$ are the case for $\alpha=0.25$. e, $\mathbf{f}$, $\mathbf{h}, \mathbf{i}$ are sample velocities and sample pathes. Red curves are the mean. $\mathbf{c}, \mathbf{I}$ are the mean and standard deviations

where $\left\langle\omega_{1}, \omega_{2}\right\rangle=\int_{0}^{T} \omega_{1}(s) \omega_{2}(s) \mathrm{d} s$. Therefore,

$$
\begin{aligned}
\left(\begin{array}{l}
\xi_{1} \\
\xi_{2}
\end{array}\right)= & \frac{1}{\left\|\omega_{1}\right\|^{2}\left\|\omega_{2}\right\|^{2}-\left(\left\langle\omega_{1}, \omega_{2}\right\rangle\right)^{2}} \\
& \times\left(\begin{array}{cc}
\left\|\omega_{2}\right\|^{2} & -\left\langle\omega_{1}, \omega_{2}\right\rangle \\
-\left\langle\omega_{1}, \omega_{2}\right\rangle & \left\|\omega_{1}\right\|^{2}
\end{array}\right) \cdot\left(\begin{array}{c}
\frac{D}{M \tau_{1} \tau_{2} \gamma} \\
\frac{D}{M \tau_{1} \tau_{2} \gamma}
\end{array}\right) .
\end{aligned}
$$

Summarize the result we have

$$
E I_{1}(v)=C_{1}(T, R) \cdot \frac{D}{M^{1-2 \alpha} \tau_{1} \tau_{2} \gamma}
$$

where

$$
C_{1}(T, R)=\int_{T}^{T+R}\left[\int_{T}^{s} b_{12}^{2}(t-s) \mathrm{d} t\right] W(s) \mathrm{d} s
$$

is a constant depending on $T$ and $R$, and

$W(t)=\frac{\omega_{1}\left\|\omega_{2}\right\|^{2}+\omega_{2}\left\|\omega_{1}\right\|^{2}-\left\langle\omega_{1}, \omega_{2}\right\rangle \cdot\left(\omega_{1}+\omega_{2}\right)}{\left\|\omega_{1}\right\|^{2}\left\|\omega_{2}\right\|^{2}-\left(\left\langle\omega_{1}, \omega_{2}\right\rangle\right)^{2}}$
It can be easily shown that $\mathrm{E} I_{1}$ converges to zero with a rate of $1 / M^{1-2 \alpha}$, similar to $\mathrm{E}_{2}$.

Numerically, $\lambda_{M}^{*}$ for $t \in[0, T]$ can be exactly constructed as $\lambda_{M}^{*}$ for $t \in[T, T+R]$ with the width of being $M$ or $-M$ depending on $t$. More precisely, for a given time step $h$ and $t+(k+1) h \leq T, k=0,1,2, \ldots$, we define

$$
\begin{aligned}
& \lambda_{M}^{*}(t) \\
& \quad= \begin{cases}\operatorname{sign}(\beta(k h)) \cdot M & \text { if } 0 \leq t-k h<h *|\beta(k h)| \\
0 & \text { if } h *|\beta(k h)| \leq t-k h<h\end{cases}
\end{aligned}
$$

\subsection{Numerical simulations}

In Fig. 2, we plot two cases of $\alpha=1$ (a, b, c, d, e, f) and $\alpha=0.25$ with $M=500(\mathrm{~g}, \mathrm{~h}, \mathrm{i}, \mathrm{j}, \mathrm{k}, \mathrm{l})$. It is clearly shown that when $M=500, \alpha=0.25$, the control accuracy is improved considerably, in comparison with the case of $\alpha=1$. This numerical simulation is in agreement with our theoretical results derived above. The parameters used are $\tau_{1}=224$ $\mathrm{ms} \tau_{2}=13 \mathrm{~ms}, T=50 \mathrm{~ms}, R=50 \mathrm{~ms}, D=10$ degree, 

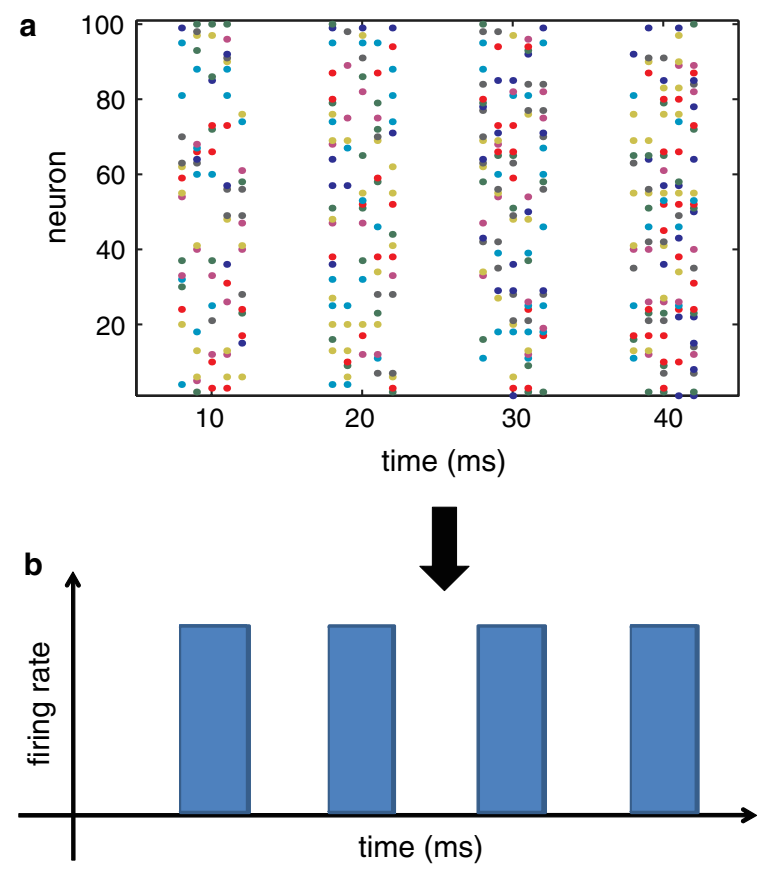

Fig. 3 Schematic plot of Young measure type control signals. A Raster plot of 100 neurons. B The firing rate of the 100 neurons, which turns out to be Young measure type signals

$\gamma=1 e-2, \kappa=0.58$, exactly the same set of parameters as in Harris and Wolpert (1998). It is interesting to compare Fig. 2d with g, for example. We see that with our approach, the accuracy is improved by 25 times! When we use $M=$ 10, an improvement of 10 times is achieved (data not shown).

\section{Discussion}

Thanks to the theoretical approach of Young measure, we demonstrate that the presence of noise in the neural control signals does not necessarily limit the precision of our movements. Our ideas originated from the analysis of a classical model of movement control (Harris and Wolpert 1998). In particular, we have observed that the solution to this problem changes qualitatively, depending on how fast the noise (its variance) scale with the signal amplitude, i.e., while in the supralinear case $(\alpha \geq 0.5)$ there is a positive lower bound on the movement error, in the sublinear case $(0<\alpha<0.5)$ such lower bound vanishes, hence we can in principle find controls which reduce the movement error arbitrarily close to zero. This abrupt transition reflects the loss of convexity of the cost functional for $\alpha<0.5$, which makes the most interesting case for our purposes.

It turns out that concentrating the control signal in two large and short pulses is not the only way to minimize the cost. The larger the pulse is, the better the control is. Also, because our control signals are effectively distributed throughout the whole duration of the movement, they are inherently more robust to perturbations. One can see that variability in the actual implementation of our control strategy have little effect on the performance.

Implement of the Young measure approach in motor control in neuroscience could be natural. Assume we have an ensemble of neurons which fire spikes within a sequence of time windows, as shown in Fig. 3A (raster plot). We see that the firing rate as plotted in Fig. 3B naturally gives us the Young measure type of control signals. Actually, the firing patterns as depicted in Fig. 3A is widely reported in experiments, see for example Rossoni et al. (2008). Besides, our approach has great potential ramifications in other fields, e.g., robotics.

Acknowledgments J. Feng was partially supported by grants from CARMEN (EPSRC, UK) and BION (EU). Jing Kang was sponsored by Institute of Advance Study Early Career Fellowship.

\section{References}

Brown D, Feng JF, Ferrick S (1999) Varibility of firing of HodgkinHuxley and FitzHuge-Nagumo neurons with stochastic input. Phys Rev Lett 82:4731-4734

Christen M, Nicol A, Kendrick K et al (2006) Odour encoding in olfactory neuronal networks beyond synchronisation. Neuroreport 17:1499-1502

Feng JF (2004) Computational neuroscience. Chapman and Hall/CRC Press, Boca Raton

Feng JF, Zhang KW (2002) Towards a mathematical foundation of minimum-variance theory. J Phys A 35:7287-7304

Feng JF, Tuckwell HC (2003) Optimal control of neuronal activity. Phys Rev Lett 91:018101

Feng JF, Tartaglia G, Tirozzi B (2004) A note on the minimum variance theory and beyond. J Phys A 37:4685-4700

Harris CM, Wolpert DM (1998) Signal-dependent noise determines motor planning. Nature 394:780-784

Hanson FB (2007) Applied stochastic processes and control for jumpdiffusions: modeling, analysis and computation, SIAM books: advances in design and control series. Cambridge University Press, Cambridge

Horton P et al (2005) Applications of multi-variate analysis of variance (MANOVA) to multi-electrode array electrophysiology data. J Neurosci Methods 146:22-41

Osborne LC, Lisberger SG, Bialek W (2005) Time course of information about motion direction in visual area MT. Nature 437:412-416

Pedregal P (1999) Optimization, telaxation and Young measures. Bull Am Math Soc 36:27-58

Robinson DA, Gordon JL, Gordon SEA (1986) A model of the smooth pursuit eye movement system. Biol Cybern 55:43-57

Rossoni E et al (2008) Synchronous bursting of oxytocin neurons; emergent behaviour of a model, PLoS Comp Biol 4(7):e1000123. doi:10.1371/journal.pcbi.1000123

Tanaka H, Krakauer J, Qian N (2006) An optimization principle for determining movement duration. J Neurophysiol 95:3875-3886

Tuckwell H (1984) Theoretical neurobiology. Cambridge University Press, Cambridge 
Valadier M (1990) Young measures, methods of nonconvex analysis. Lecture Notes in Mathematics, vol 1446. Springer, Berlin, pp 152 188

Young LC (1937) Generalized curves and the existence of an attained absolute minimum in the calculus of variations. C.R. Soc Sci Lett Vars III 30:212-234
Young LC (1942) Generalized surfaces in the calculus of variations Ann Math 43:84-103; 530-544 